\title{
Exciton optical absorption in self-similar aperiodic lattices
}

\author{
Enrique Maciá* and Francisco Domínguez-Adame ${ }^{\dagger}$ \\ Departamento de Física de Materiales, Facultad de Físicas, Universidad Complutense, E-28040 Madrid, Spain
}

(Received 5 July 1994)

\begin{abstract}
Exciton optical absorption in self-similar aperiodic one-dimensional systems is considered, focusing our attention on Thue-Morse and Fibonacci lattices as canonical examples. The absorption line shape is evaluated by solving the microscopic equations of motion of the Frenkel-exciton problem on the lattice, in which on-site energies take on two values, according to the Thue-Morse or Fibonacci sequences. Results are compared to those obtained in random lattices with the same stoichiometry and size. We find that aperiodic order causes the occurrence of well-defined characteristic features in the absorption spectra, which clearly differ from the case of random systems, indicating a most peculiar exciton dynamics. The origin of all the absorption lines is assigned by considering the self-similar aperiodic lattices as composed of two-center blocks, within the same spirit of the renormalization group ideas.
\end{abstract}

\section{INTRODUCTION}

During the past few years the notion of aperiodic order has progressively emerged to gain a proper understanding of new physical systems. Since the remarkable discovery of the quasicrystalline phase ${ }^{1}$ and the technical advances in submicrometer physics for the fabrication of semiconductor superlattices arranged according to the (quasiperiodic) Fibonacci ${ }^{2}$ and Thue-Morse ${ }^{3,4}$ sequences, much work has been devoted to the study of systems whose structural order is described by means of deterministic substitution sequences,${ }^{5}$ leading to self-similar aperiodic lattices. The interest in exploring the physical properties of elementary excitations in one-dimensional (1D) aperiodic systems, including Fibonacci, Thue-Morse, or Rudin-Shapiro lattices and their generalizations, goes beyond a formal theoretical analysis of systems deserving a simpler mathematical treatment than three-dimensional ones. In fact, it is actually well known that aperiodic order gives rise to properties which are completely absent in both periodic (crystalline) and random (amorphous) $1 \mathrm{D}$ systems. In this way, aperiodic systems exhibit highly fragmented electron ${ }^{6,7}$ and phonon ${ }^{8,9}$ spectra. These exotic electronic spectra strongly influence the transport properties, being somewhat intermediate between ballistic and difussive, which gives rise to unusual behavior of the dc conductance at finite temperature. ${ }^{10}$

In addition, it has also been realized that systems ordered according to the Fibonacci sequence exhibit some characteristic properties which are not shared by other self-similar aperiodic arrangements. Thus, from studies concerning the electronic ${ }^{1-13}$ and phonon ${ }^{14}$ properties, some authors have claimed that the kind of order associated to the Thue-Morse sequence must be considered as intermediate between the quasiperiodic order displayed by Fibonacci systems and the periodic order. Nevertheless, we feel that, in some of the above mentioned works, the criteria introduced to determine the degree of periodicity associated to a given self-similar lattice are somewhat vague and could lead to possible misinterpretations. ${ }^{15}$ Therefore, to attain a deeper insight into the nature of the order displayed by different kinds of aperiodic structures, it seems convenient to investigate transport properties different from those usually considered (electron propagation, phonon dynamics).

In this regard, optical properties of aperiodic lattices have received much less attention and, to our knowledge, most of the work has been restricted to the study of optical phenomena in Fibonacci superlattices. ${ }^{16,17}$ In this work we investigate the optical-absorption spectra of two different kinds of self-similar aperiodic systems, namely, the Fibonacci lattice (FL) and the Thue-Morse lattice (TML), and compare them with the optical spectra characteristic of both binary random and periodic related systems. To this end, we make use of a general treatment which allows us to study the dynamics of Frenkel excitons in these lattices, solve the microscopic equations of motion, and find the optical-absorption spectra. This study is inspired in our previous work showing that short-range correlated disorder has profound effects on trapping ${ }^{18}$ and optical properties ${ }^{19}$ of Frenkelexciton systems. These results lead, in a natural way, to the question as to whether long-range aperiodic order modifies exciton dynamics in comparison to long-range disorder effects. The main conclusions of this work are twofold. First, we show that both FLs and TMLs exhibit optical-absorption spectra quite different from those obtained in random and periodic lattices. Therefore optical spectra can be used to characterize experimentally the occurrence of aperiodic order in the sample. Second, we show that optical spectra are able to discriminate also the particular kind of aperiodic order present in the system. Hence the analysis of optical-absorption spectra appears as an excellent diagnostic tool to characterize the structural order from an experimental point of view.

The rest of the paper is organized as follows. In Sec. II 
we describe our model and the different self-similar aperiodic arrangements we investigate, showing how optical spectra can be numerically obtained. Section III is devoted to finding the relationship between the lines and the underlying lattice topology by means of the so-called two-center problem, guided by renormalization group ideas. Then, the origin of the lines appearing in the optical spectra of self-similar aperiodic systems is explained in Sec. IV and compared with spectra of random and periodic systems. Section V concludes the paper with a brief summary of results and some general remarks on the physical implications and possible extensions of our results.

\section{MODEL}

We consider a system of $N$ optically active centers, occupying positions in a regular $1 \mathrm{D}$ lattice with spacing unity. For our present purposes we neglect all thermal degrees of freedom, and thus we omit electron-phonon coupling and local lattice distortions. Therefore the effective Hamiltonian that describes the Frenkel-exciton problem can be written in the well-known tight-binding form with nearest-neighbor interactions as follows (we use units such that $\hbar=1$ ):

$$
\mathcal{H}=\sum_{k} V_{k} a_{k}^{\dagger} a_{k}+T \sum_{k}\left(a_{k}^{\dagger} a_{k+1}+a_{k+1}^{\dagger} a_{k}\right)
$$

Here $a_{k}^{\dagger}$ and $a_{k}$ are Bose operators creating and annihilating an electronic excitation of energy $V_{k}$ at site $k$, respectively. $T$ is the nearest-neighbor coupling, which is assumed to be constant in the whole lattice. In what follows we consider that $V_{k}$ can only take on two values, $V_{A}$ and $V_{B}$, and we shall arrange them either aperiodically, according to the Thue-Morse and Fibonacci sequences, or randomly. For convenience we define $c$ as the ratio between the number of sites $B$ and the total number of sites $N$ in the lattice.

TML and FL are canonical examples of deterministic and aperiodically ordered systems, and they can be generated by the following substitution rules: $A \rightarrow A B$, $B \rightarrow B A$ for the Thue-Morse sequence and $A \rightarrow A B$, $B \rightarrow A$ for the Fibonacci one. In this way, finite and self-similar aperiodic lattices are obtained by $n$ successive applications of the substitution rule. The $n$th generation lattice will have $2^{n}$ elements in the TML and $F_{n}$ elements for the FL, where $F_{n}$ denotes a Fibonacci number. Such numbers are generated from the recurrence law $F_{n}=F_{n-1}+F_{n-2}$ starting with $F_{0}=F_{1}=1$; as $n$ increases the ratio $F_{n-1} / F_{n}$ converges toward $\tau=(\sqrt{5}-$ $1) / 2=0.618 \ldots$, an irrational number which is known as the inverse golden mean. Therefore the on-site excitation energies are arranged according to the sequence $V_{A} V_{B} V_{B} V_{A} V_{B} \ldots$ in the TML and $V_{A} V_{B} V_{A} V_{A} V_{B} \ldots$ in the FL. The value of $c$ is strictly equal to 0.5 for any generation of the TML. On the contrary, the value of $c$ depends on the particular generation of the FL, but for large systems one has $c \sim 1-\tau$. Finally, it is worth noticing that $B$ centers appear isolated in FLs. This is an important fact in order to explain the results we will present later.

Having presented our model we now briefly describe the method we have used to calculate the absorption spectra. The line shape $I(E)$ of an optical-absorption process in which a single exciton is created in a lattice with $N$ sites can be obtained as follows. ${ }^{20}$ Let us introduce a set of correlation functions

$$
G_{k}(t)=\sum_{j}\left\langle 0\left|a_{k}(t) a_{j}^{\dagger}\right| 0\right\rangle,
$$

where $|0\rangle$ denotes the exciton vacuum state and $a_{k}(t)=$ $\exp (i \mathcal{H} t) a_{k} \exp (-i \mathcal{H} t)$ is the annihilation operator in the Heisenberg representation. The function $G_{k}(t)$ obeys the equation of motion

$$
i \frac{d}{d t} G_{k}(t)=\sum_{j} H_{k j} G_{j}(t),
$$

with the initial condition $G_{k}(0)=1$. The diagonal elements of the tridiagonal matrix $H_{k j}$ are $V_{k}$ whereas offdiagonal elements are simply given by $T$. The microscopic equation of motion is a discrete Schrödinger-like equation on a lattice and standard numerical techniques may be applied to obtain the solution. Once these equations of motion are solved, the line shape is found from the following expression:

$$
I(E)=-\frac{2}{\pi N} \int_{0}^{\infty} d t e^{-\alpha t} \sin (E t) \operatorname{Im}\left(\sum_{k} G_{k}(t)\right)
$$

where the factor $\exp (-\alpha t)$ takes into account the broadening due to the Lorentzian instrumental resolution function of width $\alpha$.

\section{THE TWO-CENTER PROBLEM}

One of the most remarkable aspects of the electronic spectra in 1D aperiodic systens is their highly fragniented nature. The detailed structure of the fragmentation pattern is mainly determined by short-range effects and it has been properly described by means of real space renormalization group concepts in the past few years. ${ }^{21,22}$ The number of subbands in the spectrum is directly related to the adopted blocking scheme which, for a binary system within the weak bound approach, usually decouples the original lattice in a series of single $(A, B)$ or double $(A A, A B, B B)$ constituent elements. Hence, from the assumption that the lattice topology must have profound influences on the exciton dynamics, it seems natural to extend the main ideas inspiring the renormalization procedure to account for the origin of the different lines appearing in the optical spectra of aperiodic systems. To 
this end, we shall consider the two-center problem describing the optical-absorption spectrum of two isolated but coupled sites, labeled 1 and 2 .

$$
\begin{aligned}
i \frac{d}{d t} G_{1}(t) & =V_{1} G_{1}(t)+T G_{2}(t), \\
i \frac{d}{d t} G_{2}(t) & =V_{2} G_{2}(t)+T G_{1}(t) .
\end{aligned}
$$

Solving these equations exactly with the initial conditions $G_{1}(0)=G_{2}(0)=1$ and inserting the result in (4) one obtains (we neglect the instrumental resolution for simplicity)

$$
I(E)=I_{+} \delta\left(E-E_{+}\right)+I_{-} \delta\left(E-E_{-}\right),
$$

where

$$
\begin{aligned}
& I_{ \pm}=\frac{1}{2} \mp \frac{1}{2}\left[1+\left(\frac{V_{1}-V_{2}}{2 T}\right)^{2}\right]^{-1 / 2} \\
& E_{ \pm}=\frac{V_{1}+V_{2}}{2} \mp T \sqrt{1+\left(\frac{V_{1}-V_{2}}{2 T}\right)^{2}}
\end{aligned}
$$

As expected, the optical-absorption spectrum of the two-center problem presents two well-defined lines. From Eqs. (6b) and (6c) we arrive at the following possible situations. If $V_{1}=V_{2}$, the intensity $I_{+}$vanishes so that spectrum exhibits a single line. Depending on the nature of the centers this line will be centered either at $E_{-}^{A A}=V_{A}+T(A A$ pairs $)$ or $E_{-}^{B B}=V_{B}+T(B B$ pairs). On the contrary, if the on-site excitation energies are different (say $V_{1}=V_{A}$ and $V_{2}=V_{B}$ ) the optical spectrum presents two components centered at $E_{ \pm}^{A B}=\left(V_{A}+V_{B}\right) / 2 \mp T \sqrt{1+\left(V_{A}-V_{B}\right)^{2} / 4 T^{2}}$. Therefore with the aid of the two-center model we can uniquely assign specific absorption lines to each of the pairs in which our original lattice can be decomposed, according to the renormalization group ideas mentioned above. In this sense, the signatures of $A A$ and $B B$ pairs are single lines located at $E_{-}^{A A}$ and $E_{-}^{B B}$. In addition, $A B$ or $B A$ pairs can be associated, irrespectively, to the simultaneous presence of two characteristic lines in the spectrum, centered at $E_{-}^{A B}$ and $E_{+}^{A B}$. In accord with these precise assignments, the origin of the main lines and satellites appearing in the absorption spectra of both FLs and TMLs can be unequivocally established.

\section{RESULTS AND DISCUSSION}

We have solved numerically the equation of motion (3) using an implicit integration scheme. In the remainder of the paper, energy will be measured in units of $T$ whereas time will be expressed in units of $T^{-1}$. Notice that $|T|$ is proportional to the exciton bandwidth so the energy scale is deduced from the experiment. Aperiodic lattices are generated using the inflation rules discussed above. We have checked that the position and the strength of all lines of the spectra are independent of the system size. Hereafter we will fix $N=2^{11}=2048$ for the TML and
$N=F_{16}=1597$ for the $\mathrm{FL}$ as representative values. In addition, random lattices with the required size $N$ and value of $c$ are generated to compare with TMLs and FLs. In order to minimize end effects, spatial periodic boundary conditions are introduced in all cases. Since we are mainly interested in characterizing the effects due to aperiodic arrangements of optical centers as compared to randomness, we will fix the values of $V_{A}, V_{B}$, and $T$. Furthermore, in order to facilitate the comparison with our previous work, we have set $V_{A}=4, V_{B}=10$, and $T=$ -1 hereafter. The width of the instrumental resolution was $\alpha=0.5$. The maximum integration time and the integration time step were 16 and $8 \times 10^{-3}$, respectively.

For the sake of clarity let us consider briefly the typical spectra associated to both pure $A$ and pure $B$ lattices corresponding to periodic cases. In the pure $A$ lattice the spectrum is a single Lorentzian line centered at $E_{\text {pure }}^{A}=V_{A}+2 T$, which with our choice of parameters is $E_{\text {pure }}^{A}=2.0$. When $B$ centers are introduced at random in the lattice, a broadening of this main line is observed accompanied by a shift of its position towards higher energies. In random systems both the broadening and the shift increase on increasing defect concentration, ${ }^{19}$ in agreement with the average- $T$-matrix approximation (ATA). ${ }^{20}$ A similar behavior takes place in the pure $B$ lattice when $A$ centers are introduced with the main difference that, in this case, the single Lorentzian absorption line is originally located at $E_{\text {pure }}^{B}=V_{B}+2 T=8.0$. Keeping these general results in mind we now proceed to discuss the main features of the spectra obtained in aperiodic systems. For convenience, we shall discuss both kinds of aperiodic lattices separately.

\section{A. Thue-Morse absorption spectrum}

In Fig. 1 we show the obtained spectrum for the TML and compare it to that corresponding to a typical random lattice. First of all, we observe the occurrence of a strong line centered at $E=2.9$ in the TML. This line is accompanied by a small shoulder at around $E=3.8$

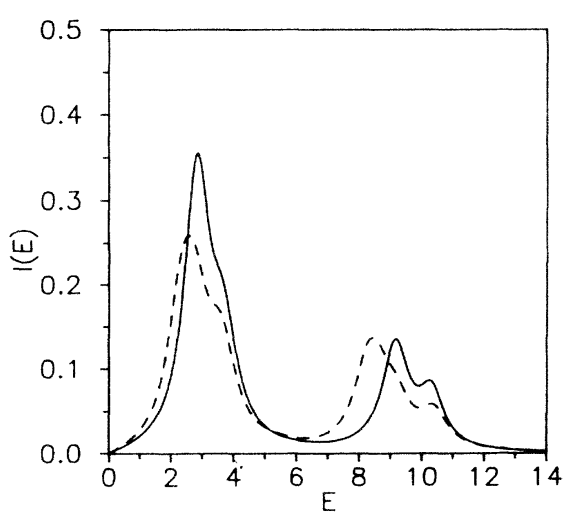

FIG. 1. Absorption spectra for a 1D Thue-Morse lattice (solid line) and a random lattice (dashed line). In both cases the system size is $N=2048$ and the concentration of $B$ centers is $c=0.5$. 
(the position of the shoulder has been obtained using two Lorentzian functions to fit data in the energy range from 0 up to 6). Moreover, two satellites appear in the highenergy region of the spectrum at about $E=9.0$ and $E=10.2$. On the other hand, concerning the random lattice, we note that the main absorption line is centered at about $E=2.6$, closer to the position corresponding to the single line in the pure $A$ lattice. In addition, the intensity of the overall absorption features in the energy range $0 \leq E \leq 6$ is smaller than those corresponding to the TML. To conclude, we observe that the random lattice also presents a characteristic pair of satellites at the high-energy region of the spectrum. One of them is centered at $E=10.2$, as occurs in the TML, but the other is found at $E=8.2$. Finally, the satellite at $E=9.0$, clearly observed in the TML, appears as an almost unnoticeable shoulder causing the asymmetry of the line at $E=8.2$.

The origin of these features can be readily accounted for making use of the expressions obtained in the twocenter problem. The main line centered at $E=2.9$ is very close to the characteristic line $E_{-}^{A A}=3.0$ associated to the $A A$ pair, hence strongly suggesting the possible origin of this absorption line. At this point it is important to note that the ATA approach cannot account for the presence of this line, since the shift of the main Lorentzian at $E_{\text {pure }}^{A}=2.0$ in the pure $A$ lattice due to the presence of $B$ centers in a concentration $c=0.5$ amounts to only 0.6 units. This value is more than $30 \%$ lower than that obtained in the TML spectrum. Thus it becomes clear that the main absorption line observed in the TML is not simply the $E_{\text {pure }}^{A}=2.0$ line shifted by the presence of $B$ centers, as occurs in the random lattice. This result suggests that the aperiodic order displayed by the TML has profound effects on the resulting exciton dynamics, which in turn manifest in the optical spectra. To find a heuristic explanation of the different exciton dynamics in TML, we would like to draw attention to the fact that the $A$ centers can appear only isolated or grouped in pairs in the TML, but never forming larger groups, as could be the case in random lattices. In fact, the presence of these larger segments, which behave locally as pure $A$ lattice segments having $B$ centers at the ends, is what explains the shift of the $E_{\text {pure }}^{A}=2.0$ line towards higher energies within the framework of ATA. Therefore the absence of large groups of $A$ centers in the TML, along with the relative abundance of $A A$ pairs instead, causes the occurrence of a noticeable and high peak at $\sim 3.0$. Similar reasoning explains the absence of the $E_{\text {pure }}^{B}=8.0$ line in the TML spectrum, whereas such a line is clearly seen in the corresponding random lattice spectrum, shifted to $E=8.2$ due to the presence of $A$ centers. Furthermore, the marked satellite at $E_{-B}^{B B}=9.0$ must be associated to the presence of many $B B$ pairs in the TML. Finally, the characteristic absorption satellites associated to $A B$ pairs are revealed as a shoulder $\left(E_{-}^{A B}=3.8\right)$ at the highenergy side of the main line and as an absorption line at $E_{+}^{A B}=10.2$. In order to further confirm this identification we have made use of Lorentzian fitting of data to evaluate the ratio between the relative intensity of lines at $E_{-}^{A B}=3.8$ and $E_{+}^{A B}=10.2$, which is found to be

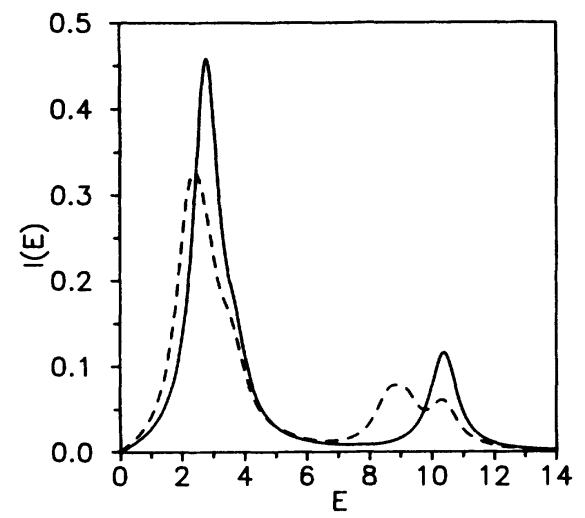

FIG. 2. Absorption spectra for a 1D Fibonacci lattice (solid line) and a random lattice (dashed line). In both cases the system size is $N=F_{16}=1597$ and the concentration of $B$ centers is $c=0.382$.

$I_{-} / I_{+} \simeq 2.04$. This value agrees rather well with the theoretical estimation $I_{-} / I_{+}=(\sqrt{10}+1) /(\sqrt{10}-1) \simeq 1.92$ obtained from Eq. (6b).

\section{B. Fibonacci absorption spectrum}

Let us now turn to the FL and compare it to a random lattice with the same size $N$ and $c$. Results are shown in Fig. 2. The FL presents two clearly distinct lines. In the low-energy range we observe a main absorption line centered at $E=2.9$ embodying an almost unobservable shoulder at $E=3.8$; meanwhile, in the high-energy region of the spectrum, a single satellite at $E=10.2$ is observed. On the other side, the spectrum associated to the random lattice shows a main absorption line at about $E=2.3$ along with a smaller shoulder at about $E=3.8$ in the low-energy region whereas, at higher energies, two broad satellites are clearly observable at $E=9.0$ and $E=10.2$. Once again the contribution due to $A A$ pairs (main peak at 2.9) and $A B$ pairs (small shoulder at $\simeq 3.8$ and satellite peak at 10.2) are clearly seen in the absorption spectrum, hence supporting the convenience of our two-center description. Moreover, one of the most remarkable characteristics of this spectrum, as compared to that corresponding to the TML, is the dramatic absence of the $E_{-}^{B B}=9.0$ line. In fact, according to our previous discussion such a line comes from the contribution of $B B$ pairs but, as is well known, such pairs are forbidden in the FL. We feel this is a very significant result since it further confirms the correctness of our interpretation about the origins of the different lines appearing in the spectra and, at the same time, allows for an easy and confident differentiaton between different kinds of aperiodic self-similar lattices from an experimental point of view.

\section{CONCLUSIONS}

In summary, we have studied the absorption spectra corresponding to the Frenkel-exciton Hamiltonian on self- 
similar aperiodic systems described by the Thue-Morse and Fibonacci sequences. By comparing the obtained spectra with those corresponding to random lattices we conclude that FLs and TMLs exhibit characteristic absorption spectra, different in many aspects from those of binary random lattices with the same stoichiometry, and that certain spectral lines can be used to characterize the aperiodic order associated to FLs from that related to TMLs. On the other side, from the viewpoint of physical applications, we have obtained analytical expressions which explain our spectra and relate microscopic system parameters such as on-site excitation energies to experimental data such as position and strengths of the lines. This relationship surely should facilitate future experimental work on optical properties of quasicrystalline solids.

Our treatment allows us to introduce, in a rather straightforward and natural way, concepts inspired in renormalizaton group techniques, which have accomplished great success in describing the electronic spectra of aperiodic systems. In the light of the obtained results and previous discussions, we think that the question as to whether Thue-Morse systems are more or less periodic than Fibonacci ones, a controversy which has raised some debate during the last few years, is still ill posed. In our opinion, both Thue-Morse and Fibonacci systems display a new kind of order, namely, self-similar aperiodic order, which has its own peculiarities, and cannot be compared with periodically ordered systems in a simple way. In fact, we have recently shown that self-similar aperiodically ordered systems are able to encode more information, in the Shannon sense, than usual periodic ones, ${ }^{23}$ thus opening a new way to answer this question. This line of reasoning may lead to a novel vision of the concept of order. Rather than thinking about different kinds of order, classified into separate categories which are compared in a quantitative way (in the sense mentioned above of a particular category being less random or more periodic than any other one), it may be more fruitful to think about different hierarchies of order. This perspective, which is inspired by the mathematical relationships between periodic, quasiperiodic, and almost periodic functions, might be of interest to those researchers working in this field.

\section{ACKNOWLEDGMENTS}

The authors thank A. Sánchez for a critical reading of the manuscript. This work was partially supported by Universidad Complutense through Project No. PR161/93-4811.
* Also at Instituto de Estudios Interdisciplinares, El Guijo, Z4 Galapagar, E-28260 Madrid, Spain. Electronic address: fimat04@emducms1.sis.ucm.es

$\dagger$ Electronic address: fimat02@emducms1.sis.ucm.es

${ }^{1}$ D. Shechtman, I. Blech, D. Gratias, and J. W. Cahn, Phys. Rev. Lett. 53, 1951 (1984).

${ }^{2}$ R. Merlin, K. Bajema, R. Clarke, F. Y. Juang, and P. K. Bhattacharya, Phys. Rev. Lett. 55, 1768 (1985).

${ }^{3}$ R. Merlin, K. Bajema, J. Nagle, and K. Ploog, J. Phys. (Paris) Colloq. 48, C5-503 (1987).

${ }^{4}$ F. Axel and H. Tarauchi, Phys. Rev. Lett. 66, 2223 (1991).

${ }^{5}$ J. Bellissard, A. Bovier, and J. M. Ghez, Rev. Math. Phys. 4, 1 (1992).

${ }^{6}$ M. Severin and R. Riklund, Phys. Rev. B 39, 10362 (1989).

${ }^{7}$ G. Y. Oh, C. S. Ryu, and M. H. Lee, J. Phys. Condens. Matter 4, 8187 (1992).

${ }^{8}$ M. Kohmoto and J. R. Banavar, Phys. Rev. B 34, 563 (1986).

9 J. X. Zhong, J. R. Yan, and J. Q. You, J. Phys. Condens. Matter 3, 5685 (1991).

${ }^{10}$ E. Maciá, F. Domínguez-Adame, and A. Sánchez, Phys. Rev. B 49, 9503 (1994).
${ }^{11}$ C. S. Ryu, G. Y. Oh, and M. H. Lee, Phys. Rev. B 48, 132 (1993).

${ }^{12}$ D. Huang, G. Gumbs, and M. Kolár, Phys. Rev. B 46, 11479 (1992).

${ }^{13}$ C. L. Roy and A. Khan, Phys. Rev. B 49, 14979 (1994).

${ }^{14}$ S. Tamura and F. Nori, Phys. Rev. B 40, 9770 (1989).

${ }^{15}$ A. Bovier and J. M. Ghez (unpublished).

${ }^{16}$ D. Tuet, M. Potemski, Y. Y. Wang, J. C. Maan, L. Tapfer, and K. Ploog, Phys. Rev. Lett. 66, 2128 (1991).

${ }^{17}$ D. Munzar, L. Bočáek, J. Humlíček, and J. Ploog, J. Phys. Condens. Matter 6, 4107 (1994).

${ }^{18}$ F. Domínguez-Adame, B. Méndez, A. Sánchez, and E. Maciá, Phys. Rev. B 49, 3839 (1994).

${ }^{19}$ F. Domínguez-Adame, E. Maciá, and A. Sánchez, Phys. Rev. B 50, 6453 (1994).

${ }^{20}$ D. L. Huber and W. Y. Ching, Phys. Rev. B 39, 8652 (1989).

${ }^{21}$ N. Niu and F. Nori, Phys. Rev. Lett. 57, 2057 (1986); Phys. Rev. B 42, 10329 (1990).

${ }^{22}$ Y. Liu and W. Sritrakool, Phys. Rev. B 43, 1110 (1991).

${ }^{23}$ E. Maciá, F. Domínguez-Adame, and A. Sánchez, Phys. Rev. E 50, 679 (1994). 